\title{
What's New
}

\section{The 5th International One Health CONGRESS}

The One Health Congress is the world's premier conference for the worldwide One Health community. One Health advocates from all over the globe will gather for 4 days of lectures, debates, workshops, and symposia. To capture the multifaceted One Health paradigm, the Congress will have distinct program tracks on One Health Science, Antimicrobial Resistance, and the Science/Policy Interface.

June 22-25, 2018, Saskatoon, Canada

https://onehealthplatform.com/international-one-

health-congress

\section{ECOHEALTH 2018 CONGRESS}

The overall theme of Ecohealth 2018 is "Environmental and Health Equity: Connecting Local Alternatives in a Global World." This theme emphasizes the need to connect local initiatives in a world with global drivers that threaten healthy ecosystems and populations, and makes a call to tackle these forces and pursue justice.

August 15-18, 2018, Cali, Colombia

http://ecohealth2018.co

\section{World Health Summit 2018}

Held once a year, the World Health Summit has grown into the world's most prominent forum for addressing global health issues. It brings together key leaders from academia, politics, civil society, and the private sector to address the most pressing health-related challenges on the planet.

October 14-16, 2018, Berlin, Germany

https://www.worldhealthsummit.org/conference.html

\section{Keystone Symposia: Framing the Response to EMERGING Virus INFECTIONS}

The key themes to be covered include the need to understand why zoonotic diseases matter, their association with agriculture, the importance of surveillance and early detection, and the difficulties of dealing with diseases that involve both medical and veterinary communities. The conference will bring together experts in virology, immunology, vaccinology, and epidemiology with those who seek to transfer knowledge between these groups, veterinarians and industry and government.

October 14-18, 2018, Hong Kong

http://www.keystonesymposia.org/18S2

\section{The 8TH InTERnational Symposium ON EMERGING Viral Diseases}

This symposium gathers reports on outstanding scientific achievements in a variety of research fields including emerging viral pathogens, viral-host interaction, antiviral immunity, and arboviruses. It will specially organize invited talks, panel discussions, and academic posters to present the latest developments in the related areas and to explore the frontiers of emerging viral diseases. This symposium will provide global researchers an open, high-quality communication platform for exchanging the state-of-theart research and developments and for strengthening collaborations and communications.

October 20-22, 2018, Wuhan, China

http://english.whiov.cas.cn/Notice2016/201805/

t20180508_192264.html 